\title{
Atomic-Resolution Composition Mapping in EDS STEM
}

\author{
Nathan R Lugg ${ }^{1}$, Akihito Kumamoto ${ }^{1}$, Ryo Ishikawa ${ }^{1}$, Bin Feng ${ }^{1}$, Gerald Kothleitner ${ }^{2,3}$, Naoya Shibata \\ and Yuichi Ikuhara ${ }^{1}$ \\ 1. Institute of Engineering Innovation, School of Engineering, The University of Tokyo, Tokyo, Japan \\ 2. Institute for Electron Microscopy and Nanoanalysis, Graz University of Technology, Graz, Austria \\ 3. Centre for Electron Microscopy, Graz, Austria
}

The properties of many modern materials and devices are determined by the local chemical composition at interfaces and defects. In grain boundaries oxygen (vacancy) and dopant content affects ionic and electrical conductivity. At herterointerfaces, the local chemistry determines how two dissimilar crystals bond on the atomic scale. Therefore, to understand how these materials behave on the atomic scale, it is important to be able to accurately map the local chemical composition on the same scale.

Energy-dispersive x-ray spectroscopy (EDS) in scanning transmission electron microscopy (STEM) uses an atomically-fine electron probe to map the chemical structure of materials at atomic resolution [1-3]. EDS STEM has had much success in analyzing chemical composition of materials at low magnification. However, at the atomic scale, the complex electron scattering makes quantitative analysis extremely difficult [4]; there is a highly non-linear relationship between the number of x-rays detected and the number of atoms the probe interacts with, making it impossible to directly relate $\mathrm{x}$-ray counts to the number or density of atoms. Several method have been proposed to overcome this: experimental data and detailed scattering simulations can be compared on an absolute scale [5,6]. Alternatively, the effects of electron scattering can be removed from experimental data by advanced deconvolution techniques [7]. However, both of these methods require knowledge of the specimen structure and composition a priori and are not particularly suitable to problems involving unknown structures. It has also been shown that tilting the specimen can minimize the effects of electron scattering and allow access to quantitative compositional information [4]. However, this comes at the expense of atomic resolution information.

Here we discuss in detail the effects and issues that electron scattering has on atomic resolution compositional mapping, using detailed scattering simulations. Using $\mathrm{SrTiO}_{3}$ as a test case, we show that the atomic-resolution 2-dimensional fractional composition maps do not, in general, reflect the correct atomic-resolution compositional information. We present both theoretical and experimental results which are in excellent agreement - that show the issues that arise at atomic resolution. Simulated results in Fig. 1 (upper panels) show the that apparent composition on the pure $\mathrm{O}$ columns and $\mathrm{TiO}$ columns deviate significantly from the ideal - in particular the Ti:O composition on the TiO column is 2:1 when the true composition is 1:1. However, such large inaccuracies can be overcome by averaging the 2dimensional compositional data along one axis (Fig. 1, lower panel; averaged over the [010] direction), essentially averaging out the strong channelling effects that occur on the atomic scale. Therefore, while 2-dimensional compositional information may not be reliable, one still has access to accurate compositional information along a particular direction. While it is unfortunate that only the averaged 1dimensional information is quantitative, this is sufficient for the compositional analysis of interfaces, where the local chemistry is extremely important. We explore this by considering the compositional analysis of a high angle grain boundary in yttria-stabilized zirconia.

Finally, we suggest some parameters for optimizing the accuracy of averaged compositional information. 
While the averaged compositional information is accurate for thin specimens, it becomes increasingly less accurate as the thickness increases (beyond 10-20nm), due to the increasingly severe effects of electron scattering. Furthermore, due to the absorption of $\mathrm{x}$-rays within the specimen, there is no significant increase in the $\mathrm{O} K$ line signal after approximately the same thickness ( 20nm). Therefore we suggest that for accurate atomic-resolution compositional analysis, specimen thicknesses of $<20 \mathrm{~nm}$ be used.

[1] AJ D’Alfonso, B Freitag, DO Klenov and LJ Allen, PRB 81 (2010), 100101.

[2] PG Kotula, DO Klenov and HS Von Harrach, Microsc Microanal 18 (2012), p. 691.

[3] G Kothleitner, MJ Neish, NR Lugg, SD Findlay, W Grogger, F Hofer, LJ Allen, PRL 112 (2014), 085501.

[4] NR Lugg, G Kothleitner, N Shibata, Y Ikuhara, Ultramicr 151 (2015), 150.

[5] HL Xin, C Dwyer and DA Muller, Ultramic 139 (2014), 38.

[6] Z Chen, AJ D’Alfonso, M Weyland, DJ Taplin, LJ Allen, SD Findlay, Ultramic 157 (2015), 21.

[7] NR Lugg, M Haruta, MJ Neish, SD Findlay, T Mizoguchi, K Kimoto and LJ Allen, APL 101 (2012), 183112.

[8] This work is partly supported by: Grant-in-Aid for Scientific Research on Innovative Areas "Nano Informatics" (Grant No. 25106003) from Japan Society for the Promotion of Science (JSPS) and "Nanotechnology Platform" (Project No. 12024046) of the Ministry of Education, Culture, Sports, Science and Technology (MEXT), Japan, Grants-in-Aid for Scientific Research (A) (15H02290) and Grant-in-Aid for Scientific Research (B) (26289234) from the JSPS.
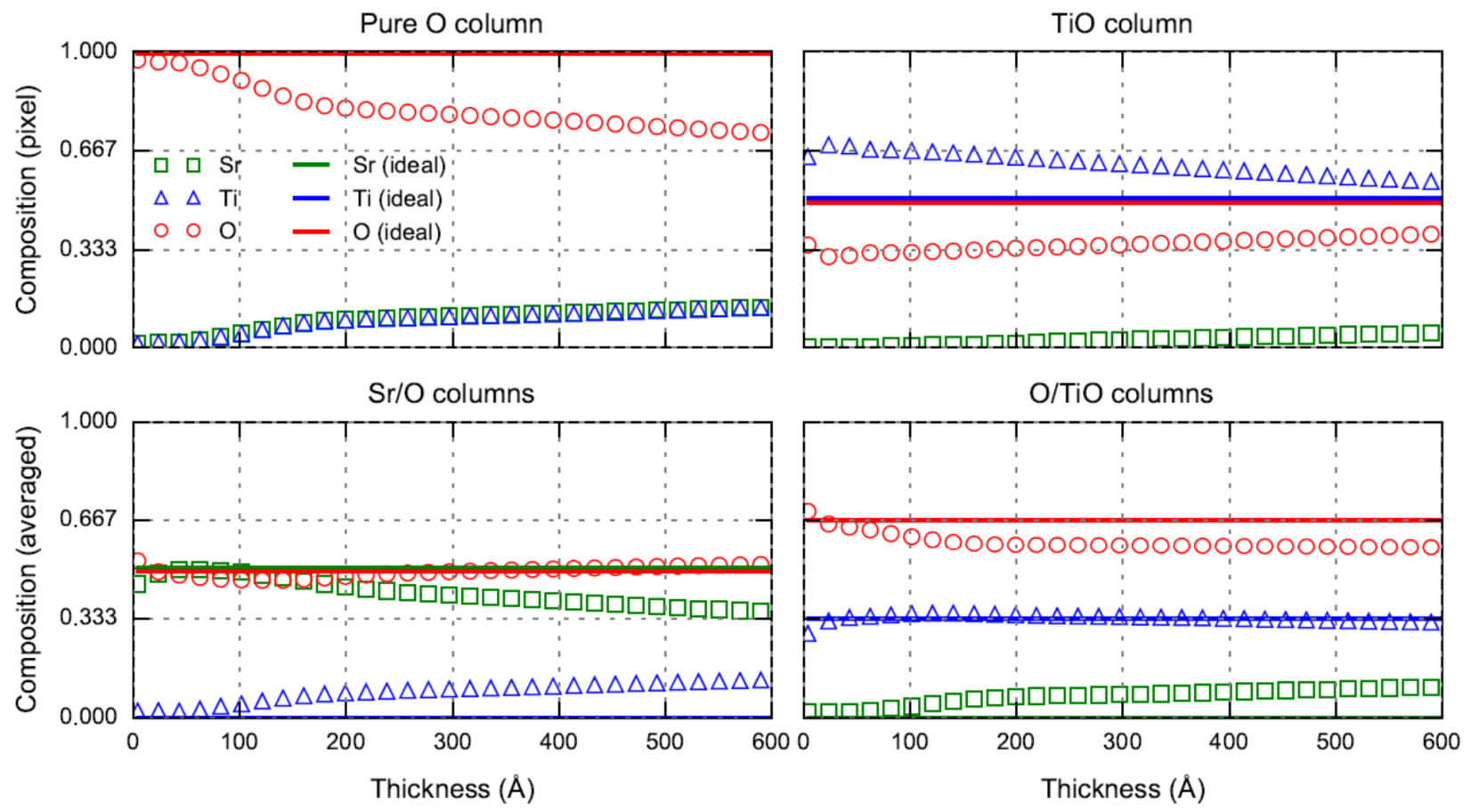

Figure 1. Simulated fractional composition of $\mathrm{Sr}, \mathrm{Ti}$ and $\mathrm{O}$ in $\mathrm{SrTiO}_{3}$. (Upper left) Composition when the probe is on a pure O column. (Upper right) Composition when the probe is on a TiO column. The lower two plots show the fractional composition after averaging the 2-dimensional fractional composition along the [010] direction. (Lower left) Composition for the (averaged) Sr/O columns. (Lower right) Composition for the (average) O/TiO columns. Solid lines show the ideal composition. 